\title{
Design and Implementation of Van de Graaff Generator
}

\author{
Aditya Jaitu ${ }^{1}$, Amish lohar ${ }^{2}$, Sumit Patil ${ }^{3}$, Vaishnavi Mokal ${ }^{4}$ \\ Prof. Nikhil Kasar ${ }^{5}$ \\ 1,2,3,4 UG Student ${ }^{5}$ Assistant Professor \\ 1,2,3,4,5 Department of Electrical Engineering \\ 1,2,3,4,5 Vishwaniketan Institude Of Engineering and Technology, Khalapur, Maharashtra, India
}

\begin{abstract}
The paper proposes a design and implementation of Van de Graaff generator whose output voltage is $80.28 \mathrm{KV}$. To calculate the generated output voltage we have use the sphere gap method. A Van de Graaff generator is an electrostatic generator as it works on a principle of electrostatics. A Van de Graaff generator is used to generate a high DC voltage. It was first developed by Robert Jemison Van de Graaff in 1929, in USA and carries his name along 1931.
\end{abstract}

This generator uses a moving belt to build up very high voltages on a hollow metal globe which is located on top of the generator. The potential difference that is developed in modern Van de Graaff generator reach about 5 megavolts. A Van de Graaff generator that we design and built that is intended to be used in schools and colleges for teaching the basics principles of Electrostatics and Electromagnetics as well as many other applications.

Nowadays many schools and colleges laboratories and many museums use this type of generators for electrostatics demonstrations.

Keywords:- Electrostatic Machine, High Voltage Generator, Van De Graaff Generator, Electrostatic Precipitator, Triboelectric Series, Electrostatic Precipitor.

\section{INTRODUCTION}

When the Greek philosopher Thales discovered that amber attracted light objects when rubbed, Electrostatics was first noticed at the times in 600 B.C. The phenomenon demonstrated a fundamental concept of electrostatics. It is a fact that extremely high voltages can be generated by friction. This fact is the basic concept, how Van de Graff generator works. This generator is named after Dr. Robert J. Van de Graaff who patented his electrostatic generator in 1935. He developed this generator for studying the acceleration of charged particles to explore the atom. The output of this device was applied in several fields of physics, astrophysics, medical and industry. In the same way is very useful in teaching corona discharge and electrostatics phenomenon.

In our project we have use single phase motor that is running our nylon belt which is held by two pully. The material use for our dome is Steel also the discharge dome is made up of steel. We give the input voltage of 9 volt Dc source and we get $80.28 \mathrm{KV}$. For calculating such a large high DC voltage we have use Sphere gap method.

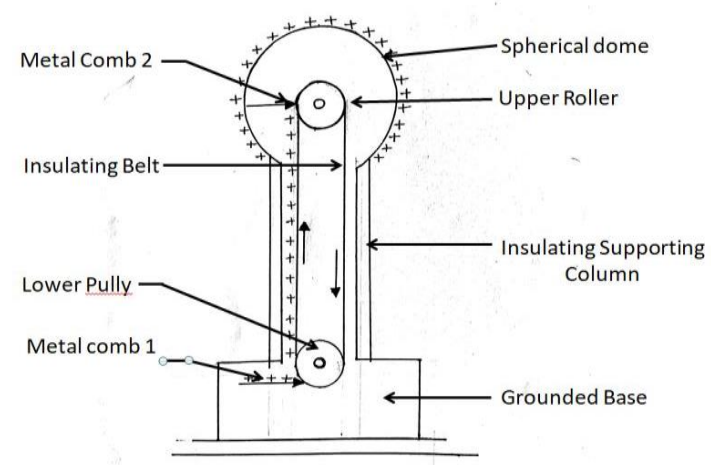

Fig 1:- Basic diagram of Van de Graaff generator

\section{LITERATURE SURVEY}

We all know Robert J. Van de graaff invented the generator known as Van de graaff generator in 1929. The generator works on the principle of electrostatics. [1]

The generator has been developed for studying the acceleration of charged particles to explore the atoms. This instrument has wide applications in science and this application was applied in several fields like physics, medicals and industry. Van de Graaff generator also has wide applications in teaching purpose in school and college laboratories to teach electrostatics principles and electromagnetics. [2]

\section{SPECIFICATIONS OF COMPONENTS USED IN VAN DE GRAAFF}

\section{> AC Motor:}

In our project we have used the $220 \mathrm{~V}$ AC sewing machine motor, which has speed of 9500 RPM. This motor is used to rotate the nylon belt with the help of two rollers.

Belt:

The belt is made up of nylon, because the nylon belt is an insulator and not a very good conductor of electricity, the positive charge does not evenly distribute over the belt. $7.5 \mathrm{~cm}$. 


\section{Insulating Supporting coloumn:}

The insulating supporting coloumn is a PVC pipe, which has diameter of $8.8 \mathrm{~cm}$ and is $65 \mathrm{~cm}$ long.

\section{Rollers:}

There are two rollers which rotates the nylon belt. The rollers we have used are basically PVC pipes, where upper roller is constant and lower roller which rotates the belt towards upper roller. And both the pipes (rollers) are 8.1 $\mathrm{cm}$ in length and diameter is $2.1 \mathrm{~cm}$.

\section{Metallic Dome:}

In our project we have used the Steel dome which works as collector. The diameter of Steel dome is $22 \mathrm{~cm}$.

\section{Battery:}

We have used 9V DC battery, which supply positive charge to nylon belt.

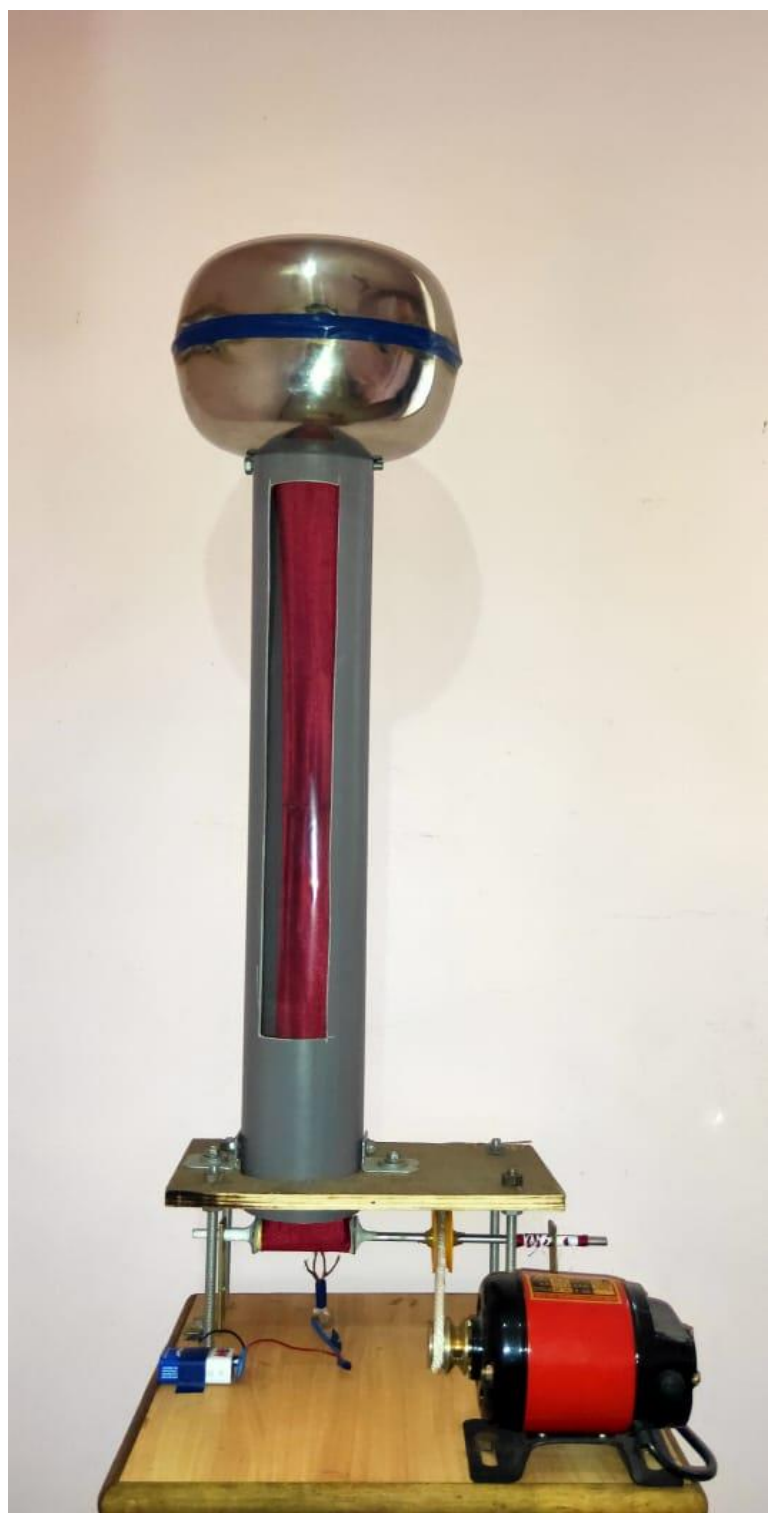

Fig 2:- Actual hardware diagram

\section{PROBLEM STATEMENT}

In our experiment there are several problems that may cause effect on the output voltage of the Van de Graaff generator i.e.

$>$ Shape and size of the steel dome.

$>$ The surrounding humidity of the Air, temperature of Air and Dust.

Material of the belt which creates the static charges.

$>$ Speed and RPM of motor which is used to rotate the belt.

$>$ Material of both rollers which is based on triboelectric series.

How these problems are improved?

The metallic dome would have perfect spherical shape and size. The output voltage depends on the humidity, dust and temperature of surrounding atmosphere. So operate this machine under proper temperature and atmosphere. The speed of the motor should be constant while rotating the belt, as no vibrations can creates effect on output voltage. The brush should have sharp edges so high amount of charges can be transferred through it.

\section{EXPECTED OUTCOMES}

We get a spark at $3 \mathrm{~cm}$, with a sound. The output voltage of Van de Graaff generator can easily determine by sphere gap method,

$$
V=\frac{2 * d * E}{\frac{d}{R}+\sqrt{\left(\frac{d}{R}\right)^{2}+4}}
$$

Where, $d=3 \mathrm{~cm}, R=11 \mathrm{~cm}$ and we can calculate $E$ as,

$$
E=27.2 * \delta\left(1+\frac{0.54}{\sqrt{\delta * R}}\right)
$$

Where, $\delta$ is called as correction factor which depends upon the temperature and pressure.

$$
\delta=\frac{293 p}{760(T+273)}
$$

By putting the value $T=30^{\circ} \mathrm{C}$ and $p=760$ torr.

$\delta=0.967$ and $E=30.657$; we get the output voltage as, $V=80.28 \mathrm{KV}$

\section{CONCLUSION}

The Van de Graaff generator in its simplest form is seen as very instructive machine, because of its solidity. This generator has become an illustrative apparatus of electrostatic influence machine.

A high voltage Van de Graaff generator is used in many schools and colleges laboratories to study the basics principles of high voltage electrostatic charge and various laws of electrostatics. The Van de Graaff generator also produces electric fields; hence the generator is very useful as those fields are strong enough too measured, operate and played with. 
Many peoples have been intensified by lightning and sparks, therefore this machine has been displayed in exhibitions, many science museums and science centres. Also by Van de Graaff generator corona discharge can be seen which occurs at about $30 \mathrm{KV}$. Some big Van de Graaff generators can produce over 200,000 to $1,000,000$ volts of high voltage lightning electrical discharge.

\section{REFFERENCES}

[1]. Museum of Science, Boston: History of the Van de Graaff generator; 1995. http://www.mos.org/sln/toe/history.html

[2]. Museum of Science, Boston: Construction of the Van de Graaff generator 1995. http://www.mos.org/sln/toe/construction.html

[3]. How the Van de Graaff generator Works. http://ffden2.phys.uaf.edu/211.fall2000.web.projects/c.reade/Van degraaff/vdghowitwrks.html

[4]. Article van de graaff generator in electrical engineering handbook.

[5]. Interactive java tutorial- Van de Graaff Generator national high magnetic field laboratory. 